\title{
Selective preparation of oxygen rich [60]fullerene derivatives by stepwise addition of $t$-butylperoxy radical and further functionalization of the fullerene mixed peroxides
}

\author{
Shaohua Huang, ${ }^{a}$ Zuo Xiao, ${ }^{a}$ Fudong Wang, ${ }^{a}$ Liangbing Gan, ${ }^{a, b}$ Xiang Zhang, ${ }^{a}$ \\ Xiangqing Hu, ${ }^{a}$ Shiwei Zhang, ${ }^{c, d}$ Mujian Lu, ${ }^{d}$ Qinqi Pan, ${ }^{d}$ Li Xu ${ }^{d, e}$
}

${ }^{a}$ Key Laboratory of Bioorganic Chemistry and Molecular Engineering of the Ministry of Education, College of Chemistry and Molecular Engineering, Peking University, Beijing 100871; ${ }^{\mathrm{b}}$ State Key Laboratory of Organometallic Chemistry, Shanghai Institute of Organic Chemistry, Chinese Academy of Sciences, 354 Fenglin Lu,

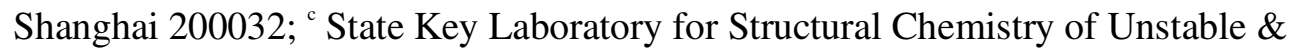
Stable Species; ${ }^{\mathrm{d}}$ College of Chemistry and Molecular Engineering, Peking University, Beijing 100871; ${ }^{\mathrm{e}}$ Beijing Institute of Microchemistry, Beijing 100091, China

\section{Table of contents}

Table 1. Crystal data and structure refinement for $\mathrm{A} .\left(\mathrm{C}_{60}(\mathrm{O})(\mathrm{OH})_{2}(\mathrm{OMe})_{4}\left(\mathrm{OOBu}^{\mathrm{t}}\right)_{2}(18)\right) \ldots . . \mathrm{S} 1$

Table 2. Atomic coordinates......S4

Table 3. Bond lengths $[\mathrm{A}]$ and angles $[\mathrm{deg}]$ for A......S7

Table 4. Anisotropic displacement parameters......S16

Table 5. Hydrogen coordinates......S19

Table 6. Hydrogen bonds......S20

ORTEPs plots......S20 
Table 1. Crystal data and structure refinement for $\mathrm{A} .\left(\mathrm{C}_{60}(\mathrm{O})(\mathrm{OH})_{2}(\mathrm{OMe})_{4}\left(\mathrm{OOBu}^{\mathrm{t}}\right)_{2}(18)\right)$

Identification code
Empirical formula
Formula weight
Temperature
Measurement device
Measurement method
Wavelength
Program for data collection
Program for cell refinement
Program for data reduction
Program for structure solution
Program for structure refinement
Program for molecular graphics
Program for publication material
Crystal system, space group
Unit cell dimensions

Volume

Refls. No. for cell measurement
Z, Calculated density

Absorption coefficient

$\mathrm{F}(000)$

Crystal shape / Crystal colour

Crystal size

Theta range for data collection

Limiting indices

Reflections collected / unique

Reflections with I>2sigma(I)

Completeness to theta $=25.00$

Decay correction (\%)

Absorption correction

Max. and min. transmission

Method for primary solution

Method for secondary solution

Hydrogen addition / treatment

Refinement method

Data / restraints / parameters

Goodness-of-fit on $\mathrm{F}^{\wedge} 2$
A C72.50 H32 O11 S

1111.04

293(2) K

Rigaku RAXIS RAPID IP

Oscillation

$0.71073 \mathrm{~A}$

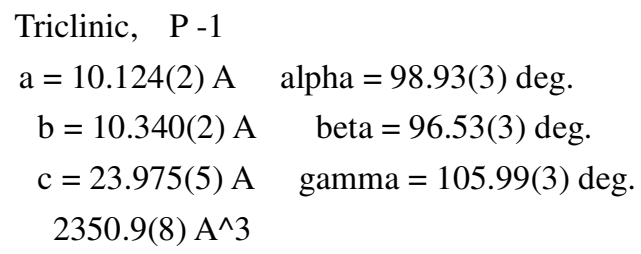

18635

2.09 to $25.00 \mathrm{deg}$.

\section{2, $\quad 1.570 \mathrm{Mg} / \mathrm{m}^{\wedge} 3$}

$0.148 \mathrm{~mm}^{\wedge}-1$

1142

Block / Red-Brown

$0.40 \times 0.30 \times 0.15 \mathrm{~mm}$

2.09 to $25.00 \mathrm{deg}$.

$-12<=\mathrm{h}<=11,-12<=\mathrm{k}<=12,-28<=\mathrm{l}<=28$

$18635 / 7914[\mathrm{R}($ int $)=0.0674]$

4003

$95.5 \%$

?

Empirical

0.978 and 0.441

direct

difmap

I

Full-matrix least-squares on $\mathrm{F}^{\wedge} 2$

7914 / 0 / 774

1.198 
Final $\mathrm{R}$ indices [ $\mathrm{I} 2 \operatorname{sigma}(\mathrm{I})]$

$\mathrm{R}$ indices (all data)

Extinction coefficient

Largest diff. peak and hole

Max. and mean shift/sigma
$\mathrm{R} 1=0.0810, \mathrm{wR} 2=0.2216$

$\mathrm{R} 1=0.1477, \mathrm{wR} 2=0.2483$

$0.0050(15)$

0.534 and -0.484 e. $\mathrm{A}^{\wedge}-3$

0.000 and 0.000 
Table 2. Atomic coordinates ( $\times 10^{\wedge} 4$ ) and equivalent isotropic displacement parameters $\left(\mathrm{A}^{\wedge} 2 \times 10^{\wedge} 3\right)$ for $\mathrm{A}$.

$\mathrm{U}(\mathrm{eq})$ is defined as one third of the trace of the orthogonalized Uij tensor.

\begin{tabular}{|c|c|c|c|c|}
\hline & $\mathrm{x}$ & $\mathrm{y}$ & $\mathrm{z}$ & $\mathrm{U}(\mathrm{eq})$ \\
\hline$S$ & $1305(3)$ & $-4219(3)$ & $-220(1)$ & $138(1)$ \\
\hline $\mathrm{O}(1)$ & $-2194(3)$ & 2499(4) & $3054(1)$ & $57(1)$ \\
\hline $\mathrm{O}(2)$ & $-3681(4)$ & $3706(5)$ & $3868(1)$ & $82(1)$ \\
\hline $\mathrm{O}(3)$ & $-3369(4)$ & $5751(4)$ & $3426(2)$ & $71(1)$ \\
\hline $\mathrm{O}(4)$ & $-4099(4)$ & $-1431(4)$ & $2698(2)$ & $83(1)$ \\
\hline $\mathrm{O}(5)$ & $-7657(5)$ & $-747(6)$ & $3443(3)$ & $124(2)$ \\
\hline$O(6)$ & $-1180(3)$ & $4655(3)$ & $2500(2)$ & $59(1)$ \\
\hline $\mathrm{O}(7)$ & $-3317(4)$ & $6688(4)$ & 1499(2) & $67(1)$ \\
\hline $\mathrm{O}(8)$ & $-7139(5)$ & $3213(8)$ & $3864(2)$ & $121(2)$ \\
\hline $\mathrm{O}(9)$ & $-6630(6)$ & $-1442(7)$ & $3555(3)$ & $150(3)$ \\
\hline $\mathrm{O}(10 \mathrm{~A})$ & $-7569(11)$ & $2356(10)$ & $4151(4)$ & $93(4)$ \\
\hline $\mathrm{O}(10 \mathrm{~B})$ & $-8271(8)$ & $3504(7)$ & $3936(3)$ & $66(3)$ \\
\hline $\mathrm{O}(11)$ & $-3946(4)$ & $427(5)$ & $3529(2)$ & $86(1)$ \\
\hline $\mathrm{C}(1)$ & $-3675(5)$ & $2238(5)$ & 2983(2) & $52(1)$ \\
\hline$C(2)$ & $-4331(5)$ & $3227(5)$ & $3291(2)$ & $56(1)$ \\
\hline $\mathrm{C}(3)$ & $-5832(5)$ & $2311(7)$ & $3227(2)$ & $63(2)$ \\
\hline $\mathrm{C}(4)$ & $-5938(5)$ & $1015(7)$ & $3085(2)$ & $63(2)$ \\
\hline $\mathrm{C}(5)$ & $-4532(5)$ & $737(5)$ & $3009(2)$ & $58(1)$ \\
\hline $\mathrm{C}(6)$ & $-2904(4)$ & $2545(5)$ & $2509(2)$ & $47(1)$ \\
\hline $\mathrm{C}(7)$ & $-2616(4)$ & $3933(5)$ & $2295(2)$ & $47(1)$ \\
\hline $\mathrm{C}(8)$ & $-2951(4)$ & $3524(5)$ & $1652(2)$ & $48(1)$ \\
\hline $\mathrm{C}(9)$ & $-3127(4)$ & $2075(5)$ & $1511(2)$ & $53(1)$ \\
\hline$C(10)$ & $-3164(5)$ & $1462(5)$ & $2010(2)$ & $53(1)$ \\
\hline$C(11)$ & $-4224(5)$ & $4611(5)$ & $3031(2)$ & $55(1)$ \\
\hline$C(12)$ & $-5636(5)$ & $4796(5)$ & $2844(2)$ & $53(1)$ \\
\hline$C(13)$ & $-5598(5)$ & $5369(5)$ & $2337(2)$ & $52(1)$ \\
\hline $\mathrm{C}(14)$ & $-4337(5)$ & $5238(4)$ & $2119(2)$ & $46(1)$ \\
\hline$C(15)$ & $-3638(5)$ & $4651(4)$ & $2469(2)$ & $47(1)$ \\
\hline$C(16)$ & $-7069(6)$ & $2855(6)$ & $3274(2)$ & $66(2)$ \\
\hline$C(17)$ & $-8374(5)$ & $1817(7)$ & $2918(2)$ & $67(2)$ \\
\hline $\mathrm{C}(18)$ & $-9053(5)$ & $2522(7)$ & $2540(2)$ & $64(2)$ \\
\hline$C(19)$ & $-8151(5)$ & $3872(6)$ & $2560(2)$ & $57(1)$ \\
\hline $\mathrm{C}(20)$ & $-6904(5)$ & $4064(6)$ & $2962(2)$ & $57(1)$ \\
\hline
\end{tabular}




\begin{tabular}{|c|c|c|c|c|}
\hline$C(21)$ & $-7286(6)$ & $-101(7)$ & $2952(3)$ & $79(2)$ \\
\hline $\mathrm{C}(22)$ & $-7317(6)$ & $-1016(6)$ & 2394(3) & $76(2)$ \\
\hline$C(23)$ & $-8569(6)$ & $-1185(6)$ & 2009(4) & $78(2)$ \\
\hline$C(24)$ & $-9271(5)$ & $-261(6)$ & $2240(3)$ & $74(2)$ \\
\hline$C(25)$ & $-8480(6)$ & $496(7)$ & 2771(3) & $73(2)$ \\
\hline$C(26)$ & $-4632(5)$ & $-388(5)$ & 2496(3) & $69(2)$ \\
\hline $\mathrm{C}(27)$ & $-6132(6)$ & $-1094(5)$ & 2185(3) & $73(2)$ \\
\hline$C(28)$ & $-6160(6)$ & $-1359(5)$ & 1594(3) & $77(2)$ \\
\hline$C(29)$ & $-4835(6)$ & $-566(5)$ & 1477(3) & $69(2)$ \\
\hline$C(30)$ & $-3988(5)$ & $144(5)$ & 1999(2) & $56(1)$ \\
\hline $\mathrm{C}(31)$ & $-9105(6)$ & 1681(7) & $615(2)$ & $73(2)$ \\
\hline $\mathrm{C}(32)$ & $-8410(6)$ & $742(7)$ & $397(2)$ & $75(2)$ \\
\hline $\mathrm{C}(33)$ & $-7080(5)$ & $1526(6)$ & $267(2)$ & $66(2)$ \\
\hline $\mathrm{C}(34)$ & $-6969(6)$ & 2977(6) & $436(2)$ & $65(2)$ \\
\hline$C(35)$ & $-8207(5)$ & $3068(6)$ & $652(2)$ & $60(1)$ \\
\hline$C(36)$ & $-9890(5)$ & $1359(6)$ & 1048(3) & $70(2)$ \\
\hline$C(37)$ & $-9786(5)$ & $2422(6)$ & $1525(3)$ & $65(2)$ \\
\hline $\mathrm{C}(38)$ & $-9851(5)$ & 1813(6) & 2041(3) & $66(2)$ \\
\hline $\mathrm{C}(39)$ & $-9980(5)$ & $384(6)$ & 1873(3) & $73(2)$ \\
\hline$C(40)$ & $-9997(5)$ & $129(7)$ & 1271(3) & $77(2)$ \\
\hline $\mathrm{C}(41)$ & $-8525(6)$ & $-453(6)$ & $594(3)$ & $75(2)$ \\
\hline$C(42)$ & $-9321(6)$ & $-804(6)$ & 1042(3) & $82(2)$ \\
\hline $\mathrm{C}(43)$ & $-8625(6)$ & $-1457(6)$ & 1427(4) & $82(2)$ \\
\hline $\mathrm{C}(44)$ & $-7331(7)$ & $-1552(6)$ & 1202(4) & $93(2)$ \\
\hline$C(45)$ & $-7303(7)$ & $-946(6)$ & $688(3)$ & $79(2)$ \\
\hline$C(46)$ & $-5936(6)$ & 1062(7) & $361(2)$ & $75(2)$ \\
\hline $\mathrm{C}(47)$ & $-6025(6)$ & $-193(6)$ & $585(3)$ & $75(2)$ \\
\hline$C(48)$ & $-4766(6)$ & $-14(7)$ & $965(3)$ & $75(2)$ \\
\hline $\mathrm{C}(49)$ & $-3880(5)$ & 1374(6) & $998(2)$ & $61(1)$ \\
\hline$C(50)$ & $-4584(5)$ & $2020(6)$ & $620(2)$ & $63(2)$ \\
\hline $\mathrm{C}(51)$ & $-5691(6)$ & $3877(6)$ & $687(2)$ & $62(1)$ \\
\hline$C(52)$ & $-4464(5)$ & $3408(6)$ & $775(2)$ & $57(1)$ \\
\hline$C(53)$ & $-3603(5)$ & $4159(5)$ & 1293(2) & $49(1)$ \\
\hline $\mathrm{C}(54)$ & $-4153(5)$ & $5345(5)$ & 1517(2) & $51(1)$ \\
\hline$C(55)$ & $-5607(5)$ & 4931(5) & $1137(2)$ & $51(1)$ \\
\hline$C(56)$ & $-8113(5)$ & $4045(6)$ & 1098(2) & $55(1)$ \\
\hline $\mathrm{C}(57)$ & $-6796(5)$ & $5003(5)$ & 1349(2) & $53(1)$ \\
\hline $\mathrm{C}(58)$ & $-6811(5)$ & $5251(5)$ & $1965(2)$ & $54(1)$ \\
\hline $\mathrm{C}(59)$ & $-8089(5)$ & $4489(5)$ & 2084(2) & $53(1)$ \\
\hline$C(60)$ & $-8936(5)$ & $3723(6)$ & 1554(2) & $59(1)$ \\
\hline$C(61)$ & $-3118(6)$ & 6992(6) & $960(3)$ & $85(2)$ \\
\hline$C(62)$ & $-4049(8)$ & 2931(10) & 4303(3) & $123(3)$ \\
\hline$C(63)$ & $-706(5)$ & $5956(6)$ & 2354(3) & $87(2)$ \\
\hline$C(64)$ & $-2636(6)$ & $-1168(6)$ & 2753(3) & $91(2)$ \\
\hline
\end{tabular}




\begin{tabular}{lclll}
$\mathrm{C}(65)$ & $-6875(11)$ & $-2083(8)$ & $4027(3)$ & $109(3)$ \\
$\mathrm{C}(66)$ & $-8246(17)$ & $-2790(20)$ & $4082(9)$ & $307(13)$ \\
$\mathrm{C}(67)$ & $-5735(10)$ & $-2724(9)$ & $4116(4)$ & $124(3)$ \\
$\mathrm{C}(68)$ & $-6810(30)$ & $-870(20)$ & $4520(5)$ & $348(15)$ \\
$\mathrm{C}(69)$ & $-8978(15)$ & $2730(9)$ & $4382(5)$ & $177(6)$ \\
$\mathrm{C}(70)$ & $-10057(13)$ & $3535(13)$ & $4415(5)$ & $174(4)$ \\
$\mathrm{C}(71)$ & $-8012(12)$ & $3290(20)$ & $4917(4)$ & $260(10)$ \\
$\mathrm{C}(72)$ & $-9853(13)$ & $1465(16)$ & $4105(5)$ & $198(6)$ \\
$\mathrm{C}(73)$ & 0 & -5000 & 0 & $91(3)$ \\
\hline
\end{tabular}


Table 3. Bond lengths [A] and angles [deg] for A.

\begin{tabular}{|c|c|}
\hline S-C(73) & $1.543(2)$ \\
\hline $\mathrm{O}(1)-\mathrm{C}(6)$ & $1.432(6)$ \\
\hline $\mathrm{O}(1)-\mathrm{C}(1)$ & $1.434(5)$ \\
\hline $\mathrm{O}(2)-\mathrm{C}(2)$ & $1.411(6)$ \\
\hline $\mathrm{O}(2)-\mathrm{C}(62)$ & $1.431(8)$ \\
\hline $\mathrm{O}(3)-\mathrm{C}(11)$ & $1.393(6)$ \\
\hline $\mathrm{O}(4)-\mathrm{C}(64)$ & $1.417(7)$ \\
\hline $\mathrm{O}(4)-\mathrm{C}(26)$ & $1.453(6)$ \\
\hline $\mathrm{O}(5)-\mathrm{O}(9)$ & $1.441(7)$ \\
\hline $\mathrm{O}(5)-\mathrm{C}(21)$ & $1.476(7)$ \\
\hline $\mathrm{O}(6)-\mathrm{C}(63)$ & $1.411(6)$ \\
\hline $\mathrm{O}(6)-\mathrm{C}(7)$ & $1.428(5)$ \\
\hline $\mathrm{O}(7)-\mathrm{C}(61)$ & $1.400(6)$ \\
\hline $\mathrm{O}(7)-\mathrm{C}(54)$ & $1.424(6)$ \\
\hline $\mathrm{O}(8)-\mathrm{O}(10 \mathrm{~A})$ & $1.222(9)$ \\
\hline $\mathrm{O}(8)-\mathrm{O}(10 \mathrm{~B})$ & $1.285(8)$ \\
\hline $\mathrm{O}(8)-\mathrm{C}(16)$ & $1.422(7)$ \\
\hline $\mathrm{O}(9)-\mathrm{C}(65)$ & $1.409(8)$ \\
\hline $\mathrm{O}(10 \mathrm{~A})-\mathrm{O}(10 \mathrm{~B})$ & $1.659(11)$ \\
\hline $\mathrm{O}(10 \mathrm{~A})-\mathrm{C}(69)$ & $1.706(14)$ \\
\hline $\mathrm{O}(10 \mathrm{~B})-\mathrm{C}(69)$ & $1.555(10)$ \\
\hline $\mathrm{O}(11)-\mathrm{C}(5)$ & $1.437(6)$ \\
\hline$C(1)-C(6)$ & $1.478(7)$ \\
\hline$C(1)-C(2)$ & $1.512(7)$ \\
\hline$C(1)-C(5)$ & $1.570(7)$ \\
\hline$C(2)-C(3)$ & $1.529(7)$ \\
\hline$C(2)-C(11)$ & $1.629(7)$ \\
\hline$C(3)-C(4)$ & $1.302(8)$ \\
\hline$C(3)-C(16)$ & $1.515(7)$ \\
\hline$C(4)-C(21)$ & $1.488(8)$ \\
\hline$C(4)-C(5)$ & $1.553(7)$ \\
\hline$C(5)-C(26)$ & $1.529(8)$ \\
\hline$C(6)-C(10)$ & $1.450(7)$ \\
\hline$C(6)-C(7)$ & $1.562(6)$ \\
\hline$C(7)-C(15)$ & $1.490(6)$ \\
\hline $\mathrm{C}(7)-\mathrm{C}(8)$ & $1.507(7)$ \\
\hline $\mathrm{C}(8)-\mathrm{C}(53)$ & $1.376(6)$ \\
\hline $\mathrm{C}(8)-\mathrm{C}(9)$ & $1.439(7)$ \\
\hline $\mathrm{C}(9)-\mathrm{C}(49)$ & $1.351(7)$ \\
\hline$C(9)-C(10)$ & $1.438(7)$ \\
\hline
\end{tabular}




\begin{tabular}{|c|c|}
\hline$C(10)-C(30)$ & $1.382(7)$ \\
\hline $\mathrm{C}(11)-\mathrm{C}(12)$ & $1.521(7)$ \\
\hline $\mathrm{C}(11)-\mathrm{C}(15)$ & $1.535(7)$ \\
\hline$C(12)-C(20)$ & $1.384(7)$ \\
\hline $\mathrm{C}(12)-\mathrm{C}(13)$ & $1.434(7)$ \\
\hline $\mathrm{C}(13)-\mathrm{C}(58)$ & $1.396(7)$ \\
\hline $\mathrm{C}(13)-\mathrm{C}(14)$ & $1.464(6)$ \\
\hline$C(14)-C(15)$ & $1.353(6)$ \\
\hline $\mathrm{C}(14)-\mathrm{C}(54)$ & $1.495(6)$ \\
\hline$C(16)-C(17)$ & $1.517(8)$ \\
\hline$C(16)-C(20)$ & $1.534(7)$ \\
\hline $\mathrm{C}(17)-\mathrm{C}(25)$ & $1.328(8)$ \\
\hline $\mathrm{C}(17)-\mathrm{C}(18)$ & $1.473(8)$ \\
\hline $\mathrm{C}(18)-\mathrm{C}(38)$ & $1.343(8)$ \\
\hline $\mathrm{C}(18)-\mathrm{C}(19)$ & $1.432(8)$ \\
\hline $\mathrm{C}(19)-\mathrm{C}(59)$ & $1.390(7)$ \\
\hline$C(19)-C(20)$ & $1.446(7)$ \\
\hline $\mathrm{C}(21)-\mathrm{C}(22)$ & $1.505(9)$ \\
\hline $\mathrm{C}(21)-\mathrm{C}(25)$ & $1.551(8)$ \\
\hline$C(22)-C(27)$ & $1.368(8)$ \\
\hline$C(22)-C(23)$ & $1.432(8)$ \\
\hline $\mathrm{C}(23)-\mathrm{C}(43)$ & $1.371(9)$ \\
\hline $\mathrm{C}(23)-\mathrm{C}(24)$ & $1.423(9)$ \\
\hline $\mathrm{C}(24)-\mathrm{C}(25)$ & $1.410(9)$ \\
\hline $\mathrm{C}(24)-\mathrm{C}(39)$ & $1.426(8)$ \\
\hline$C(26)-C(30)$ & $1.527(8)$ \\
\hline $\mathrm{C}(26)-\mathrm{C}(27)$ & $1.536(8)$ \\
\hline $\mathrm{C}(27)-\mathrm{C}(28)$ & $1.398(9)$ \\
\hline $\mathrm{C}(28)-\mathrm{C}(44)$ & $1.372(9)$ \\
\hline $\mathrm{C}(28)-\mathrm{C}(29)$ & $1.450(8)$ \\
\hline $\mathrm{C}(29)-\mathrm{C}(30)$ & $1.402(8)$ \\
\hline $\mathrm{C}(29)-\mathrm{C}(48)$ & $1.433(9)$ \\
\hline $\mathrm{C}(31)-\mathrm{C}(36)$ & $1.403(8)$ \\
\hline $\mathrm{C}(31)-\mathrm{C}(32)$ & $1.419(8)$ \\
\hline $\mathrm{C}(31)-\mathrm{C}(35)$ & $1.454(8)$ \\
\hline $\mathrm{C}(32)-\mathrm{C}(41)$ & 1.371(9) \\
\hline $\mathrm{C}(32)-\mathrm{C}(33)$ & $1.462(8)$ \\
\hline $\mathrm{C}(33)-\mathrm{C}(46)$ & $1.380(8)$ \\
\hline $\mathrm{C}(33)-\mathrm{C}(34)$ & $1.461(8)$ \\
\hline$C(34)-C(51)$ & $1.374(7)$ \\
\hline $\mathrm{C}(34)-\mathrm{C}(35)$ & $1.430(7)$ \\
\hline$C(35)-C(56)$ & $1.327(7)$ \\
\hline$C(36)-C(37)$ & $1.431(8)$ \\
\hline $\mathrm{C}(36)-\mathrm{C}(40)$ & $1.436(8)$ \\
\hline
\end{tabular}




\begin{tabular}{|c|c|}
\hline $\mathrm{C}(37)-\mathrm{C}(60)$ & $1.370(7)$ \\
\hline $\mathrm{C}(37)-\mathrm{C}(38)$ & $1.472(8)$ \\
\hline $\mathrm{C}(38)-\mathrm{C}(39)$ & $1.435(8)$ \\
\hline$C(39)-C(40)$ & $1.424(9)$ \\
\hline $\mathrm{C}(40)-\mathrm{C}(42)$ & $1.408(9)$ \\
\hline $\mathrm{C}(41)-\mathrm{C}(42)$ & $1.445(9)$ \\
\hline $\mathrm{C}(41)-\mathrm{C}(45)$ & $1.469(9)$ \\
\hline $\mathrm{C}(42)-\mathrm{C}(43)$ & $1.454(9)$ \\
\hline C(43)-C(44) & $1.493(9)$ \\
\hline $\mathrm{C}(44)-\mathrm{C}(45)$ & $1.468(10)$ \\
\hline $\mathrm{C}(45)-\mathrm{C}(47)$ & $1.385(8)$ \\
\hline$C(46)-C(50)$ & $1.453(8)$ \\
\hline $\mathrm{C}(46)-\mathrm{C}(47)$ & $1.463(9)$ \\
\hline $\mathrm{C}(47)-\mathrm{C}(48)$ & $1.428(8)$ \\
\hline $\mathrm{C}(48)-\mathrm{C}(49)$ & $1.453(8)$ \\
\hline $\mathrm{C}(49)-\mathrm{C}(50)$ & $1.440(8)$ \\
\hline $\mathrm{C}(50)-\mathrm{C}(52)$ & $1.393(8)$ \\
\hline $\mathrm{C}(51)-\mathrm{C}(55)$ & $1.385(7)$ \\
\hline $\mathrm{C}(51)-\mathrm{C}(52)$ & $1.459(7)$ \\
\hline $\mathrm{C}(52)-\mathrm{C}(53)$ & $1.410(7)$ \\
\hline $\mathrm{C}(53)-\mathrm{C}(54)$ & $1.529(7)$ \\
\hline $\mathrm{C}(54)-\mathrm{C}(55)$ & $1.551(7)$ \\
\hline $\mathrm{C}(55)-\mathrm{C}(57)$ & $1.375(7)$ \\
\hline$C(56)-C(57)$ & $1.424(7)$ \\
\hline $\mathrm{C}(56)-\mathrm{C}(60)$ & $1.473(7)$ \\
\hline $\mathrm{C}(57)-\mathrm{C}(58)$ & $1.463(7)$ \\
\hline $\mathrm{C}(58)-\mathrm{C}(59)$ & $1.403(7)$ \\
\hline $\mathrm{C}(59)-\mathrm{C}(60)$ & $1.430(7)$ \\
\hline $\mathrm{C}(65)-\mathrm{C}(66)$ & $1.413(16)$ \\
\hline $\mathrm{C}(65)-\mathrm{C}(67)$ & $1.492(11)$ \\
\hline $\mathrm{C}(65)-\mathrm{C}(68)$ & $1.565(15)$ \\
\hline $\mathrm{C}(69)-\mathrm{C}(72)$ & $1.377(14)$ \\
\hline $\mathrm{C}(69)-\mathrm{C}(71)$ & $1.452(17)$ \\
\hline $\mathrm{C}(69)-\mathrm{C}(70)$ & $1.547(16)$ \\
\hline $\mathrm{C}(73)-\mathrm{S} \# 1$ & $1.543(2)$ \\
\hline $\mathrm{C}(6)-\mathrm{O}(1)-\mathrm{C}(1)$ & $62.1(3)$ \\
\hline $\mathrm{C}(2)-\mathrm{O}(2)-\mathrm{C}(62)$ & $121.9(5)$ \\
\hline $\mathrm{C}(64)-\mathrm{O}(4)-\mathrm{C}(26)$ & $115.4(4)$ \\
\hline $\mathrm{O}(9)-\mathrm{O}(5)-\mathrm{C}(21)$ & $105.2(4)$ \\
\hline $\mathrm{C}(63)-\mathrm{O}(6)-\mathrm{C}(7)$ & $115.3(4)$ \\
\hline $\mathrm{C}(61)-\mathrm{O}(7)-\mathrm{C}(54)$ & $117.4(4)$ \\
\hline $\mathrm{O}(10 \mathrm{~A})-\mathrm{O}(8)-\mathrm{O}(10 \mathrm{~B})$ & $82.8(6)$ \\
\hline $\mathrm{O}(10 \mathrm{~A})-\mathrm{O}(8)-\mathrm{C}(16)$ & $122.6(8)$ \\
\hline
\end{tabular}




\begin{tabular}{|c|c|}
\hline $\mathrm{O}(10 \mathrm{~B})-\mathrm{O}(8)-\mathrm{C}(16)$ & $111.5(5)$ \\
\hline $\mathrm{C}(65)-\mathrm{O}(9)-\mathrm{O}(5)$ & $110.0(6)$ \\
\hline $\mathrm{O}(8)-\mathrm{O}(10 \mathrm{~A})-\mathrm{O}(10 \mathrm{~B})$ & $50.3(4)$ \\
\hline $\mathrm{O}(8)-\mathrm{O}(10 \mathrm{~A})-\mathrm{C}(69)$ & $104.7(7)$ \\
\hline $\mathrm{O}(10 \mathrm{~B})-\mathrm{O}(10 \mathrm{~A})-\mathrm{C}(69)$ & $55.0(5)$ \\
\hline $\mathrm{O}(8)-\mathrm{O}(10 \mathrm{~B})-\mathrm{C}(69)$ & $110.4(8)$ \\
\hline $\mathrm{O}(8)-\mathrm{O}(10 \mathrm{~B})-\mathrm{O}(10 \mathrm{~A})$ & $46.9(4)$ \\
\hline $\mathrm{C}(69)-\mathrm{O}(10 \mathrm{~B})-\mathrm{O}(10 \mathrm{~A})$ & $64.0(7)$ \\
\hline $\mathrm{O}(1)-\mathrm{C}(1)-\mathrm{C}(6)$ & $58.9(3)$ \\
\hline $\mathrm{O}(1)-\mathrm{C}(1)-\mathrm{C}(2)$ & $121.7(4)$ \\
\hline $\mathrm{C}(6)-\mathrm{C}(1)-\mathrm{C}(2)$ & $122.6(4)$ \\
\hline $\mathrm{O}(1)-\mathrm{C}(1)-\mathrm{C}(5)$ & $115.2(4)$ \\
\hline $\mathrm{C}(6)-\mathrm{C}(1)-\mathrm{C}(5)$ & $121.9(4)$ \\
\hline $\mathrm{C}(2)-\mathrm{C}(1)-\mathrm{C}(5)$ & $108.7(4)$ \\
\hline $\mathrm{O}(2)-\mathrm{C}(2)-\mathrm{C}(1)$ & 111.1(4) \\
\hline $\mathrm{O}(2)-\mathrm{C}(2)-\mathrm{C}(3)$ & $112.9(4)$ \\
\hline $\mathrm{C}(1)-\mathrm{C}(2)-\mathrm{C}(3)$ & $100.7(4)$ \\
\hline $\mathrm{O}(2)-\mathrm{C}(2)-\mathrm{C}(11)$ & $104.8(4)$ \\
\hline $\mathrm{C}(1)-\mathrm{C}(2)-\mathrm{C}(11)$ & $115.0(4)$ \\
\hline $\mathrm{C}(3)-\mathrm{C}(2)-\mathrm{C}(11)$ & $112.6(4)$ \\
\hline $\mathrm{C}(4)-\mathrm{C}(3)-\mathrm{C}(16)$ & $123.8(5)$ \\
\hline $\mathrm{C}(4)-\mathrm{C}(3)-\mathrm{C}(2)$ & $112.2(5)$ \\
\hline$C(16)-C(3)-C(2)$ & $123.8(5)$ \\
\hline $\mathrm{C}(3)-\mathrm{C}(4)-\mathrm{C}(21)$ & $123.9(5)$ \\
\hline $\mathrm{C}(3)-\mathrm{C}(4)-\mathrm{C}(5)$ & $113.7(5)$ \\
\hline $\mathrm{C}(21)-\mathrm{C}(4)-\mathrm{C}(5)$ & $122.2(5)$ \\
\hline $\mathrm{O}(11)-\mathrm{C}(5)-\mathrm{C}(26)$ & $110.7(4)$ \\
\hline $\mathrm{O}(11)-\mathrm{C}(5)-\mathrm{C}(4)$ & $109.4(4)$ \\
\hline $\mathrm{C}(26)-\mathrm{C}(5)-\mathrm{C}(4)$ & 114.1(4) \\
\hline $\mathrm{O}(11)-\mathrm{C}(5)-\mathrm{C}(1)$ & $107.0(4)$ \\
\hline $\mathrm{C}(26)-\mathrm{C}(5)-\mathrm{C}(1)$ & $117.0(4)$ \\
\hline $\mathrm{C}(4)-\mathrm{C}(5)-\mathrm{C}(1)$ & $97.7(4)$ \\
\hline $\mathrm{O}(1)-\mathrm{C}(6)-\mathrm{C}(10)$ & $122.8(4)$ \\
\hline $\mathrm{O}(1)-\mathrm{C}(6)-\mathrm{C}(1)$ & $59.0(3)$ \\
\hline$C(10)-C(6)-C(1)$ & 117.7(4) \\
\hline $\mathrm{O}(1)-\mathrm{C}(6)-\mathrm{C}(7)$ & $119.6(4)$ \\
\hline$C(10)-C(6)-C(7)$ & $107.4(4)$ \\
\hline $\mathrm{C}(1)-\mathrm{C}(6)-\mathrm{C}(7)$ & $124.1(4)$ \\
\hline $\mathrm{O}(6)-\mathrm{C}(7)-\mathrm{C}(15)$ & $116.4(4)$ \\
\hline $\mathrm{O}(6)-\mathrm{C}(7)-\mathrm{C}(8)$ & $113.0(4)$ \\
\hline $\mathrm{C}(15)-\mathrm{C}(7)-\mathrm{C}(8)$ & $106.0(4)$ \\
\hline $\mathrm{O}(6)-\mathrm{C}(7)-\mathrm{C}(6)$ & $106.3(3)$ \\
\hline$C(15)-C(7)-C(6)$ & $109.9(4)$ \\
\hline $\mathrm{C}(8)-\mathrm{C}(7)-\mathrm{C}(6)$ & $104.6(4)$ \\
\hline
\end{tabular}




$\begin{array}{lr}\mathrm{C}(53)-\mathrm{C}(8)-\mathrm{C}(9) & 121.2(4) \\ \mathrm{C}(53)-\mathrm{C}(8)-\mathrm{C}(7) & 125.6(4) \\ \mathrm{C}(9)-\mathrm{C}(8)-\mathrm{C}(7) & 106.9(4) \\ \mathrm{C}(49)-\mathrm{C}(9)-\mathrm{C}(10) & 121.4(5) \\ \mathrm{C}(49)-\mathrm{C}(9)-\mathrm{C}(8) & 117.9(5) \\ \mathrm{C}(10)-\mathrm{C}(9)-\mathrm{C}(8) & 112.0(4) \\ \mathrm{C}(30)-\mathrm{C}(10)-\mathrm{C}(9) & 122.5(5) \\ \mathrm{C}(30)-\mathrm{C}(10)-\mathrm{C}(6) & 121.5(5) \\ \mathrm{C}(9)-\mathrm{C}(10)-\mathrm{C}(6) & 108.1(4) \\ \mathrm{O}(3)-\mathrm{C}(11)-\mathrm{C}(12) & 111.2(4) \\ \mathrm{O}(3)-\mathrm{C}(11)-\mathrm{C}(15) & 106.5(4) \\ \mathrm{C}(12)-\mathrm{C}(11)-\mathrm{C}(15) & 99.7(4) \\ \mathrm{O}(3)-\mathrm{C}(11)-\mathrm{C}(2) & 109.8(4) \\ \mathrm{C}(12)-\mathrm{C}(11)-\mathrm{C}(2) & 113.3(4) \\ \mathrm{C}(15)-\mathrm{C}(11)-\mathrm{C}(2) & 115.9(4) \\ \mathrm{C}(20)-\mathrm{C}(12)-\mathrm{C}(13) & 119.3(4) \\ \mathrm{C}(20)-\mathrm{C}(12)-\mathrm{C}(11) & 126.5(5) \\ \mathrm{C}(13)-\mathrm{C}(12)-\mathrm{C}(11) & 109.3(4) \\ \mathrm{C}(58)-\mathrm{C}(13)-\mathrm{C}(12) & 121.9(4) \\ \mathrm{C}(58)-\mathrm{C}(13)-\mathrm{C}(14) & 121.3(4) \\ \mathrm{C}(12)-\mathrm{C}(13)-\mathrm{C}(14) & 106.5(4) \\ \mathrm{C}(15)-\mathrm{C}(14)-\mathrm{C}(13) & 110.0(4) \\ \mathrm{C}(15)-\mathrm{C}(14)-\mathrm{C}(54) & 126.0(4) \\ \mathrm{C}(13)-\mathrm{C}(14)-\mathrm{C}(54) & 121.5(4) \\ \mathrm{C}(14)-\mathrm{C}(15)-\mathrm{C}(7) & 124.5(4) \\ \mathrm{C}(14)-\mathrm{C}(15)-\mathrm{C}(11) & 110.4(4) \\ \mathrm{C}(7)-\mathrm{C}(15)-\mathrm{C}(11) & 124.3(4) \\ \mathrm{O}(8)-\mathrm{C}(16)-\mathrm{C}(3) & 108.0(5) \\ \mathrm{O}(8)-\mathrm{C}(16)-\mathrm{C}(17) & 114.8(4) \\ \mathrm{C}(3)-\mathrm{C}(16)-\mathrm{C}(17) & 109.8(5) \\ \mathrm{O}(8)-\mathrm{C}(16)-\mathrm{C}(20) & 113.3(5) \\ \mathrm{C}(3)-\mathrm{C}(16)-\mathrm{C}(20) & 108.8(4) \\ \mathrm{C}(17)-\mathrm{C}(16)-\mathrm{C}(20) & 101.9(4) \\ \mathrm{C}(25)-\mathrm{C}(17)-\mathrm{C}(18) & 121.8(6) \\ \mathrm{C}(25)-\mathrm{C}(17)-\mathrm{C}(16) & 122.3(5) \\ \mathrm{C}(18)-\mathrm{C}(17)-\mathrm{C}(16) & 108.3(5) \\ \mathrm{C}(38)-\mathrm{C}(18)-\mathrm{C}(19) & 119.0(5) \\ \mathrm{C}(38)-\mathrm{C}(18)-\mathrm{C}(17) & 119.9(6) \\ \mathrm{C}(19)-\mathrm{C}(18)-\mathrm{C}(17) & \\ \mathrm{C}(59)-\mathrm{C}(19)-\mathrm{C}(18) & \end{array}$




\begin{tabular}{|c|c|}
\hline$C(19)-C(20)-C(16)$ & $110.6(5)$ \\
\hline $\mathrm{O}(5)-\mathrm{C}(21)-\mathrm{C}(4)$ & $113.7(6)$ \\
\hline $\mathrm{O}(5)-\mathrm{C}(21)-\mathrm{C}(22)$ & $118.3(5)$ \\
\hline $\mathrm{C}(4)-\mathrm{C}(21)-\mathrm{C}(22)$ & $108.3(5)$ \\
\hline $\mathrm{O}(5)-\mathrm{C}(21)-\mathrm{C}(25)$ & $106.3(4)$ \\
\hline$C(4)-C(21)-C(25)$ & $109.3(5)$ \\
\hline$C(22)-C(21)-C(25)$ & $99.8(5)$ \\
\hline $\mathrm{C}(27)-\mathrm{C}(22)-\mathrm{C}(23)$ & $120.2(7)$ \\
\hline $\mathrm{C}(27)-\mathrm{C}(22)-\mathrm{C}(21)$ & $122.6(6)$ \\
\hline $\mathrm{C}(23)-\mathrm{C}(22)-\mathrm{C}(21)$ & $110.1(6)$ \\
\hline $\mathrm{C}(43)-\mathrm{C}(23)-\mathrm{C}(24)$ & $118.1(6)$ \\
\hline $\mathrm{C}(43)-\mathrm{C}(23)-\mathrm{C}(22)$ & $121.4(6)$ \\
\hline $\mathrm{C}(24)-\mathrm{C}(23)-\mathrm{C}(22)$ & $109.5(6)$ \\
\hline$C(25)-C(24)-C(23)$ & $108.0(6)$ \\
\hline $\mathrm{C}(25)-\mathrm{C}(24)-\mathrm{C}(39)$ & $120.0(6)$ \\
\hline $\mathrm{C}(23)-\mathrm{C}(24)-\mathrm{C}(39)$ & $120.4(6)$ \\
\hline$C(17)-C(25)-C(24)$ & $119.4(6)$ \\
\hline$C(17)-C(25)-C(21)$ & $122.6(6)$ \\
\hline$C(24)-C(25)-C(21)$ & $110.6(6)$ \\
\hline $\mathrm{O}(4)-\mathrm{C}(26)-\mathrm{C}(30)$ & $114.2(5)$ \\
\hline $\mathrm{O}(4)-\mathrm{C}(26)-\mathrm{C}(5)$ & $108.7(5)$ \\
\hline$C(30)-C(26)-C(5)$ & $114.0(4)$ \\
\hline $\mathrm{O}(4)-\mathrm{C}(26)-\mathrm{C}(27)$ & $107.6(4)$ \\
\hline$C(30)-C(26)-C(27)$ & $98.9(5)$ \\
\hline$C(5)-C(26)-C(27)$ & $113.0(5)$ \\
\hline $\mathrm{C}(22)-\mathrm{C}(27)-\mathrm{C}(28)$ & $119.4(6)$ \\
\hline$C(22)-C(27)-C(26)$ & $126.0(7)$ \\
\hline $\mathrm{C}(28)-\mathrm{C}(27)-\mathrm{C}(26)$ & $110.2(5)$ \\
\hline $\mathrm{C}(44)-\mathrm{C}(28)-\mathrm{C}(27)$ & $123.2(6)$ \\
\hline $\mathrm{C}(44)-\mathrm{C}(28)-\mathrm{C}(29)$ & $118.0(7)$ \\
\hline $\mathrm{C}(27)-\mathrm{C}(28)-\mathrm{C}(29)$ & $108.0(5)$ \\
\hline $\mathrm{C}(30)-\mathrm{C}(29)-\mathrm{C}(48)$ & $122.1(5)$ \\
\hline $\mathrm{C}(30)-\mathrm{C}(29)-\mathrm{C}(28)$ & $108.5(6)$ \\
\hline $\mathrm{C}(48)-\mathrm{C}(29)-\mathrm{C}(28)$ & $120.3(6)$ \\
\hline$C(10)-C(30)-C(29)$ & $116.6(5)$ \\
\hline$C(10)-C(30)-C(26)$ & $126.4(5)$ \\
\hline$C(29)-C(30)-C(26)$ & $110.2(5)$ \\
\hline $\mathrm{C}(36)-\mathrm{C}(31)-\mathrm{C}(32)$ & $117.7(6)$ \\
\hline $\mathrm{C}(36)-\mathrm{C}(31)-\mathrm{C}(35)$ & $120.6(5)$ \\
\hline $\mathrm{C}(32)-\mathrm{C}(31)-\mathrm{C}(35)$ & $108.5(5)$ \\
\hline $\mathrm{C}(41)-\mathrm{C}(32)-\mathrm{C}(31)$ & $121.2(6)$ \\
\hline $\mathrm{C}(41)-\mathrm{C}(32)-\mathrm{C}(33)$ & $120.5(6)$ \\
\hline $\mathrm{C}(31)-\mathrm{C}(32)-\mathrm{C}(33)$ & $108.2(6)$ \\
\hline $\mathrm{C}(46)-\mathrm{C}(33)-\mathrm{C}(34)$ & $121.1(5)$ \\
\hline
\end{tabular}




$\begin{array}{ll}\mathrm{C}(46)-\mathrm{C}(33)-\mathrm{C}(32) & 118.9(6) \\ \mathrm{C}(34)-\mathrm{C}(33)-\mathrm{C}(32) & 107.0(5) \\ \mathrm{C}(51)-\mathrm{C}(34)-\mathrm{C}(35) & 120.9(5) \\ \mathrm{C}(51)-\mathrm{C}(34)-\mathrm{C}(33) & 118.4(5) \\ \mathrm{C}(35)-\mathrm{C}(34)-\mathrm{C}(33) & 108.1(5) \\ \mathrm{C}(56)-\mathrm{C}(35)-\mathrm{C}(34) & 119.2(5) \\ \mathrm{C}(56)-\mathrm{C}(35)-\mathrm{C}(31) & 120.4(5) \\ \mathrm{C}(34)-\mathrm{C}(35)-\mathrm{C}(31) & 108.1(5) \\ \mathrm{C}(31)-\mathrm{C}(36)-\mathrm{C}(37) & 118.6(5) \\ \mathrm{C}(31)-\mathrm{C}(36)-\mathrm{C}(40) & 122.1(6) \\ \mathrm{C}(37)-\mathrm{C}(36)-\mathrm{C}(40) & 106.6(6) \\ \mathrm{C}(60)-\mathrm{C}(37)-\mathrm{C}(36) & 120.2(5) \\ \mathrm{C}(60)-\mathrm{C}(37)-\mathrm{C}(38) & 120.3(5) \\ \mathrm{C}(36)-\mathrm{C}(37)-\mathrm{C}(38) & 107.9(5) \\ \mathrm{C}(18)-\mathrm{C}(38)-\mathrm{C}(39) & 119.2(5) \\ \mathrm{C}(18)-\mathrm{C}(38)-\mathrm{C}(37) & 120.0(5) \\ \mathrm{C}(39)-\mathrm{C}(38)-\mathrm{C}(37) & 108.0(6) \\ \mathrm{C}(40)-\mathrm{C}(39)-\mathrm{C}(24) & 121.2(6) \\ \mathrm{C}(40)-\mathrm{C}(39)-\mathrm{C}(38) & 106.7(6) \\ \mathrm{C}(24)-\mathrm{C}(39)-\mathrm{C}(38) & 119.6(6) \\ \mathrm{C}(42)-\mathrm{C}(40)-\mathrm{C}(39) & 118.4(6) \\ \mathrm{C}(42)-\mathrm{C}(40)-\mathrm{C}(36) & 119.4(6) \\ \mathrm{C}(39)-\mathrm{C}(40)-\mathrm{C}(36) & 110.9(6) \\ \mathrm{C}(32)-\mathrm{C}(41)-\mathrm{C}(42) & 122.1(6) \\ \mathrm{C}(32)-\mathrm{C}(41)-\mathrm{C}(45) & 120.2(6) \\ \mathrm{C}(42)-\mathrm{C}(41)-\mathrm{C}(45) & 106.3(6) \\ \mathrm{C}(40)-\mathrm{C}(42)-\mathrm{C}(41) & 117.6(6) \\ \mathrm{C}(40)-\mathrm{C}(42)-\mathrm{C}(43) & 119.1(7) \\ \mathrm{C}(41)-\mathrm{C}(42)-\mathrm{C}(43) & 110.9(6) \\ \mathrm{C}(23)-\mathrm{C}(43)-\mathrm{C}(42) & 122.8(6) \\ \mathrm{C}(23)-\mathrm{C}(43)-\mathrm{C}(44) & 118.1(6) \\ \mathrm{C}(42)-\mathrm{C}(43)-\mathrm{C}(44) & 106.6(7)-\mathrm{C}(47)-\mathrm{C}(48) \\ \mathrm{C}(28)-\mathrm{C}(44)-\mathrm{C}(45) & 122.9(6) \\ \mathrm{C}(48)-\mathrm{C}(47)-\mathrm{C}(46) & 117.7(8) \\ \mathrm{C}(28)-\mathrm{C}(44)-\mathrm{C}(43) & 106.8(6) \\ \mathrm{C}(45)-\mathrm{C}(44)-\mathrm{C}(43) & 118.1(6) \\ \mathrm{C}(47)-\mathrm{C}(45)-\mathrm{C}(44) & \\ \mathrm{C}(47)-\mathrm{C}(45)-\mathrm{C}(41) & \end{array}$




$\begin{array}{ll}\mathrm{C}(47)-\mathrm{C}(48)-\mathrm{C}(29) & 119.4(6) \\ \mathrm{C}(47)-\mathrm{C}(48)-\mathrm{C}(49) & 107.4(6) \\ \mathrm{C}(29)-\mathrm{C}(48)-\mathrm{C}(49) & 119.0(5) \\ \mathrm{C}(9)-\mathrm{C}(49)-\mathrm{C}(50) & 121.5(5) \\ \mathrm{C}(9)-\mathrm{C}(49)-\mathrm{C}(48) & 118.1(5) \\ \mathrm{C}(50)-\mathrm{C}(49)-\mathrm{C}(48) & 108.3(5) \\ \mathrm{C}(52)-\mathrm{C}(50)-\mathrm{C}(49) & 120.0(5) \\ \mathrm{C}(52)-\mathrm{C}(50)-\mathrm{C}(46) & 119.0(5) \\ \mathrm{C}(49)-\mathrm{C}(50)-\mathrm{C}(46) & 108.6(5) \\ \mathrm{C}(34)-\mathrm{C}(51)-\mathrm{C}(55) & 119.6(5) \\ \mathrm{C}(34)-\mathrm{C}(51)-\mathrm{C}(52) & 121.1(5) \\ \mathrm{C}(55)-\mathrm{C}(51)-\mathrm{C}(52) & 109.2(5) \\ \mathrm{C}(50)-\mathrm{C}(52)-\mathrm{C}(53) & 118.4(5) \\ \mathrm{C}(50)-\mathrm{C}(52)-\mathrm{C}(51) & 120.0(5) \\ \mathrm{C}(53)-\mathrm{C}(52)-\mathrm{C}(51) & 109.6(4) \\ \mathrm{C}(8)-\mathrm{C}(53)-\mathrm{C}(52) & 120.8(4) \\ \mathrm{C}(8)-\mathrm{C}(53)-\mathrm{C}(54) & 122.5(4) \\ \mathrm{C}(52)-\mathrm{C}(53)-\mathrm{C}(54) & 108.5(4) \\ \mathrm{O}(7)-\mathrm{C}(54)-\mathrm{C}(14) & 110.0(4) \\ \mathrm{O}(7)-\mathrm{C}(54)-\mathrm{C}(53) & 116.6(4) \\ \mathrm{C}(14)-\mathrm{C}(54)-\mathrm{C}(53) & 105.9(4) \\ \mathrm{O}(7)-\mathrm{C}(54)-\mathrm{C}(55) & 113.0(4) \\ \mathrm{C}(14)-\mathrm{C}(54)-\mathrm{C}(55) & 109.1(4) \\ \mathrm{C}(53)-\mathrm{C}(54)-\mathrm{C}(55) & 101.6(4) \\ \mathrm{C}(57)-\mathrm{C}(55)-\mathrm{C}(51) & 119.2(5) \\ \mathrm{C}(57)-\mathrm{C}(55)-\mathrm{C}(54) & 123.2(4) \\ \mathrm{C}(51)-\mathrm{C}(55)-\mathrm{C}(54) & 109.2(4) \\ \mathrm{C}(35)-\mathrm{C}(56)-\mathrm{C}(57) & 120.1(5) \\ \mathrm{C}(35)-\mathrm{C}(56)-\mathrm{C}(60) & 119.7(5) \\ \mathrm{C}(57)-\mathrm{C}(56)-\mathrm{C}(60) & 108.8(5) \\ \mathrm{C}(55)-\mathrm{C}(57)-\mathrm{C}(56) & 120.8(5) \\ \mathrm{C}(55)-\mathrm{C}(57)-\mathrm{C}(58) & 121.2(4) \\ \mathrm{C}(56)-\mathrm{C}(57)-\mathrm{C}(58) & 105.6(4) \\ \mathrm{C}(13)-\mathrm{C}(58)-\mathrm{C}(59) & 118.3(4) \\ \mathrm{C}(13)-\mathrm{C}(58)-\mathrm{C}(57) & 118.5(4) \\ \mathrm{C}(59)-\mathrm{C}(58)-\mathrm{C}(57) & 110.4(4) \\ \mathrm{C}(19)-\mathrm{C}(59)-\mathrm{C}(58) & 121.0(4) \\ \mathrm{C}(19)-\mathrm{C}(59)-\mathrm{C}(60) & 118.2(5) \\ \mathrm{C}(56)-\mathrm{C}(59)-\mathrm{C}(60) & \end{array}$




$\begin{array}{lc}\mathrm{C}(66)-\mathrm{C}(65)-\mathrm{C}(67) & 119.9(9) \\ \mathrm{O}(9)-\mathrm{C}(65)-\mathrm{C}(68) & 103.6(9) \\ \mathrm{C}(66)-\mathrm{C}(65)-\mathrm{C}(68) & 91.6(14) \\ \mathrm{C}(67)-\mathrm{C}(65)-\mathrm{C}(68) & 114.5(11) \\ \mathrm{C}(72)-\mathrm{C}(69)-\mathrm{C}(71) & 136.8(14) \\ \mathrm{C}(72)-\mathrm{C}(69)-\mathrm{C}(70) & 99.1(13) \\ \mathrm{C}(71)-\mathrm{C}(69)-\mathrm{C}(70) & 104.4(8) \\ \mathrm{C}(72)-\mathrm{C}(69)-\mathrm{O}(10 \mathrm{~B}) & 109.3(9) \\ \mathrm{C}(71)-\mathrm{C}(69)-\mathrm{O}(10 \mathrm{~B}) & 105.1(9) \\ \mathrm{C}(70)-\mathrm{C}(69)-\mathrm{O}(10 \mathrm{~B}) & 93.2(9) \\ \mathrm{C}(72)-\mathrm{C}(69)-\mathrm{O}(10 \mathrm{~A}) & 91.7(8) \\ \mathrm{C}(71)-\mathrm{C}(69)-\mathrm{O}(10 \mathrm{~A}) & 82.6(10) \\ \mathrm{C}(70)-\mathrm{C}(69)-\mathrm{O}(10 \mathrm{~A}) & 154.1(9) \\ \mathrm{O}(10 \mathrm{~B})-\mathrm{C}(69)-\mathrm{O}(10 \mathrm{~A}) & 60.9(5) \\ \mathrm{S}-\mathrm{C}(73)-\mathrm{S} \# 1 & 180.00(19) \\ \end{array}$

Symmetry transformations used to generate equivalent atoms:

\#1 -x,-y-1,-z 
Table 4. Anisotropic displacement parameters $\left(A^{\wedge} 2 \times 10^{\wedge} 3\right)$ for $A$.

The anisotropic displacement factor exponent takes the form:

$-2 \mathrm{pi}^{\wedge} 2\left[\mathrm{~h}^{\wedge} 2 \mathrm{a}^{* \wedge} 2 \mathrm{U} 11+\ldots+2 \mathrm{~h} \mathrm{k} \mathrm{a}^{*} \mathrm{~b}^{*} \mathrm{U} 12\right]$

\begin{tabular}{|c|c|c|c|c|c|c|}
\hline & U11 & $\mathrm{U} 22$ & U33 & $\mathrm{U} 23$ & U13 & U12 \\
\hline$S$ & 111(2) & $167(2)$ & $174(2)$ & $82(2)$ & $54(2)$ & $65(2)$ \\
\hline $\mathrm{O}(1)$ & $40(2)$ & $70(2)$ & $65(2)$ & $18(2)$ & $1(2)$ & $22(2)$ \\
\hline $\mathrm{O}(2)$ & $81(3)$ & $124(3)$ & $43(2)$ & $18(2)$ & $2(2)$ & $37(2)$ \\
\hline $\mathrm{O}(3)$ & $72(2)$ & $65(2)$ & $62(2)$ & $-7(2)$ & $-5(2)$ & $15(2)$ \\
\hline $\mathrm{O}(4)$ & $60(2)$ & $61(2)$ & $140(4)$ & $45(2)$ & $12(2)$ & $28(2)$ \\
\hline $\mathrm{O}(5)$ & $92(3)$ & $139(4)$ & 194(5) & $114(4)$ & $63(4)$ & $61(3)$ \\
\hline $\mathrm{O}(6)$ & $35(2)$ & $56(2)$ & $86(2)$ & $13(2)$ & $8(2)$ & $15(2)$ \\
\hline $\mathrm{O}(7)$ & $67(2)$ & $59(2)$ & $76(2)$ & $29(2)$ & $16(2)$ & $12(2)$ \\
\hline $\mathrm{O}(8)$ & $77(3)$ & $234(7)$ & $56(3)$ & $30(4)$ & $25(2)$ & $46(4)$ \\
\hline $\mathrm{O}(9)$ & $122(4)$ & 184(6) & $203(6)$ & $124(5)$ & $85(4)$ & $72(4)$ \\
\hline $\mathrm{O}(10 \mathrm{~A})$ & $136(8)$ & $100(7)$ & $84(6)$ & $44(5)$ & $64(6)$ & $71(6)$ \\
\hline $\mathrm{O}(10 \mathrm{~B})$ & $78(6)$ & $74(5)$ & $62(5)$ & $19(4)$ & $31(4)$ & $39(4)$ \\
\hline $\mathrm{O}(11)$ & $67(2)$ & 109(3) & $105(3)$ & $70(3)$ & $13(2)$ & $34(2)$ \\
\hline $\mathrm{C}(1)$ & $41(3)$ & $62(3)$ & $56(3)$ & $20(3)$ & $2(2)$ & $17(2)$ \\
\hline $\mathrm{C}(2)$ & $50(3)$ & $71(3)$ & $50(3)$ & $16(3)$ & $4(2)$ & $25(3)$ \\
\hline $\mathrm{C}(3)$ & $59(3)$ & $87(4)$ & $61(3)$ & $40(3)$ & $20(3)$ & $33(3)$ \\
\hline $\mathrm{C}(4)$ & $48(3)$ & $92(4)$ & $65(3)$ & $48(3)$ & $19(2)$ & $26(3)$ \\
\hline $\mathrm{C}(5)$ & $52(3)$ & $62(3)$ & $70(3)$ & $32(3)$ & $11(2)$ & $23(3)$ \\
\hline$C(6)$ & $35(2)$ & $51(3)$ & $55(3)$ & $6(2)$ & $5(2)$ & $18(2)$ \\
\hline$C(7)$ & $33(2)$ & $48(3)$ & $60(3)$ & $11(2)$ & $5(2)$ & $14(2)$ \\
\hline $\mathrm{C}(8)$ & $37(2)$ & $50(3)$ & $58(3)$ & $10(2)$ & $14(2)$ & $13(2)$ \\
\hline $\mathrm{C}(9)$ & $31(2)$ & $65(3)$ & $68(3)$ & $14(3)$ & $20(2)$ & $20(2)$ \\
\hline$C(10)$ & $39(3)$ & $54(3)$ & $73(3)$ & $19(3)$ & $14(2)$ & $21(2)$ \\
\hline $\mathrm{C}(11)$ & $55(3)$ & $59(3)$ & $51(3)$ & $2(3)$ & $5(2)$ & $24(3)$ \\
\hline$C(12)$ & $58(3)$ & $58(3)$ & $46(3)$ & $-1(2)$ & $6(2)$ & $30(3)$ \\
\hline$C(13)$ & $53(3)$ & $48(3)$ & $60(3)$ & $5(2)$ & $11(2)$ & $25(2)$ \\
\hline$C(14)$ & $40(2)$ & $35(2)$ & $59(3)$ & $7(2)$ & $6(2)$ & $11(2)$ \\
\hline$C(15)$ & $42(3)$ & $40(3)$ & $55(3)$ & $8(2)$ & $4(2)$ & $11(2)$ \\
\hline$C(16)$ & $64(3)$ & $105(4)$ & $49(3)$ & $31(3)$ & $23(3)$ & $42(3)$ \\
\hline$C(17)$ & $51(3)$ & $100(5)$ & $69(3)$ & $36(4)$ & $30(3)$ & $34(3)$ \\
\hline$C(18)$ & $37(3)$ & $100(5)$ & $70(4)$ & $31(3)$ & $20(3)$ & $31(3)$ \\
\hline $\mathrm{C}(19)$ & $46(3)$ & $77(4)$ & $60(3)$ & $12(3)$ & $20(2)$ & $36(3)$ \\
\hline$C(20)$ & $53(3)$ & $74(4)$ & $51(3)$ & $1(3)$ & $14(2)$ & $33(3)$ \\
\hline $\mathrm{C}(21)$ & $61(4)$ & $83(4)$ & $119(5)$ & $71(4)$ & $35(3)$ & $32(3)$ \\
\hline
\end{tabular}




\begin{tabular}{|c|c|c|c|c|c|c|}
\hline$C(22)$ & $47(3)$ & $58(3)$ & $132(6)$ & $46(4)$ & 18(3) & $16(3)$ \\
\hline$C(23)$ & $41(3)$ & $47(3)$ & $136(6)$ & $25(4)$ & $3(4)$ & $0(3)$ \\
\hline$C(24)$ & $35(3)$ & $75(4)$ & $112(5)$ & $39(4)$ & $15(3)$ & $6(3)$ \\
\hline$C(25)$ & $46(3)$ & $91(5)$ & $99(5)$ & $50(4)$ & $33(3)$ & $24(3)$ \\
\hline$C(26)$ & $50(3)$ & $44(3)$ & $122(5)$ & $39(3)$ & $15(3)$ & $18(2)$ \\
\hline $\mathrm{C}(27)$ & $60(4)$ & $45(3)$ & $117(6)$ & $30(3)$ & $12(4)$ & $17(3)$ \\
\hline$C(28)$ & $58(4)$ & $43(3)$ & $121(6)$ & $4(3)$ & $-3(4)$ & $18(3)$ \\
\hline$C(29)$ & $53(3)$ & $45(3)$ & $111(5)$ & $0(3)$ & 13(3) & $27(3)$ \\
\hline $\mathrm{C}(30)$ & $51(3)$ & $42(3)$ & 79(4) & $3(3)$ & $6(3)$ & $28(2)$ \\
\hline$C(31)$ & $55(3)$ & $99(5)$ & $62(3)$ & $6(3)$ & $-8(3)$ & $32(3)$ \\
\hline$C(32)$ & $57(4)$ & $85(5)$ & $62(3)$ & $-16(3)$ & $-18(3)$ & $13(3)$ \\
\hline $\mathrm{C}(33)$ & $53(3)$ & $94(4)$ & $43(3)$ & $-6(3)$ & $-6(2)$ & $27(3)$ \\
\hline$C(34)$ & $70(4)$ & $79(4)$ & $48(3)$ & $10(3)$ & $0(3)$ & $30(3)$ \\
\hline$C(35)$ & $53(3)$ & $76(4)$ & $57(3)$ & 17(3) & $-1(2)$ & $32(3)$ \\
\hline$C(36)$ & $36(3)$ & $72(4)$ & $92(4)$ & $12(4)$ & $-6(3)$ & $9(3)$ \\
\hline $\mathrm{C}(37)$ & $27(3)$ & $89(4)$ & $83(4)$ & 21(4) & $5(2)$ & $23(3)$ \\
\hline $\mathrm{C}(38)$ & 29(3) & $83(4)$ & $97(5)$ & $29(4)$ & 19(3) & $24(3)$ \\
\hline $\mathrm{C}(39)$ & $22(2)$ & $73(4)$ & $119(5)$ & $24(4)$ & $8(3)$ & $5(2)$ \\
\hline $\mathrm{C}(40)$ & $41(3)$ & $81(4)$ & $95(5)$ & $6(4)$ & $-7(3)$ & 11(3) \\
\hline $\mathrm{C}(41)$ & $52(3)$ & $64(4)$ & $86(4)$ & $-14(3)$ & $-5(3)$ & 1(3) \\
\hline$C(42)$ & $48(3)$ & $57(4)$ & $119(5)$ & $-8(4)$ & $-13(4)$ & 2(3) \\
\hline $\mathrm{C}(43)$ & $53(4)$ & $44(3)$ & 131(6) & $7(4)$ & $-3(4)$ & $-2(3)$ \\
\hline$C(44)$ & $63(4)$ & $40(3)$ & $150(7)$ & $-25(4)$ & $-4(4)$ & 4(3) \\
\hline$C(45)$ & $77(5)$ & $51(3)$ & $90(5)$ & $-19(3)$ & $-4(3)$ & $13(3)$ \\
\hline$C(46)$ & $77(4)$ & $86(5)$ & $53(3)$ & $-21(3)$ & $13(3)$ & $29(4)$ \\
\hline $\mathrm{C}(47)$ & $65(4)$ & $63(4)$ & $78(4)$ & $-34(3)$ & $2(3)$ & $19(3)$ \\
\hline $\mathrm{C}(48)$ & 61(4) & $80(4)$ & $86(4)$ & $-17(4)$ & $17(3)$ & $41(3)$ \\
\hline $\mathrm{C}(49)$ & $51(3)$ & $67(4)$ & $63(3)$ & $-11(3)$ & $17(3)$ & $23(3)$ \\
\hline$C(50)$ & $52(3)$ & $90(4)$ & $48(3)$ & $-1(3)$ & $14(2)$ & $29(3)$ \\
\hline $\mathrm{C}(51)$ & $61(3)$ & $80(4)$ & $55(3)$ & $26(3)$ & $17(3)$ & 28(3) \\
\hline $\mathrm{C}(52)$ & $52(3)$ & $70(4)$ & $53(3)$ & 13(3) & $15(2)$ & 23(3) \\
\hline$C(53)$ & $42(3)$ & $53(3)$ & $49(3)$ & $10(2)$ & $12(2)$ & $11(2)$ \\
\hline $\mathrm{C}(54)$ & $50(3)$ & $45(3)$ & $62(3)$ & 19(2) & $13(2)$ & 13(2) \\
\hline $\mathrm{C}(55)$ & $53(3)$ & $60(3)$ & $51(3)$ & $25(3)$ & $12(2)$ & $27(2)$ \\
\hline$C(56)$ & $45(3)$ & $65(3)$ & $62(3)$ & $16(3)$ & $-1(2)$ & $28(3)$ \\
\hline $\mathrm{C}(57)$ & $55(3)$ & $58(3)$ & $58(3)$ & $22(3)$ & $8(2)$ & $30(3)$ \\
\hline $\mathrm{C}(58)$ & $58(3)$ & $48(3)$ & $62(3)$ & $5(2)$ & $7(3)$ & $31(2)$ \\
\hline $\mathrm{C}(59)$ & $45(3)$ & $63(3)$ & $62(3)$ & 11(3) & $12(2)$ & $35(2)$ \\
\hline$C(60)$ & 41(3) & $82(4)$ & 71(3) & 31(3) & $15(3)$ & $37(3)$ \\
\hline$C(61)$ & $80(4)$ & $85(4)$ & $103(5)$ & 49(4) & $37(4)$ & $18(3)$ \\
\hline$C(62)$ & $116(6)$ & 183(9) & $59(4)$ & $40(5)$ & $-13(4)$ & $27(6)$ \\
\hline$C(63)$ & $38(3)$ & $53(3)$ & $166(7)$ & $37(4)$ & $-2(3)$ & $3(3)$ \\
\hline$C(64)$ & $70(4)$ & $64(4)$ & $146(6)$ & $25(4)$ & $-3(4)$ & $35(3)$ \\
\hline $\mathrm{C}(65)$ & 197(9) & $81(5)$ & $66(4)$ & $30(4)$ & $45(5)$ & $50(5)$ \\
\hline
\end{tabular}




\begin{tabular}{lllllll}
$\mathrm{C}(66)$ & $259(17)$ & $380(30)$ & $440(30)$ & $340(30)$ & $229(19)$ & $148(17)$ \\
$\mathrm{C}(67)$ & $156(8)$ & $134(7)$ & $112(6)$ & $35(5)$ & $21(5)$ & $86(6)$ \\
$\mathrm{C}(68)$ & $740(50)$ & $270(20)$ & $104(9)$ & $35(11)$ & $14(16)$ & $290(30)$ \\
$\mathrm{C}(69)$ & $271(13)$ & $73(5)$ & $171(10)$ & $-14(6)$ & $191(11)$ & $-13(7)$ \\
$\mathrm{C}(70)$ & $189(11)$ & $211(12)$ & $147(9)$ & $32(9)$ & $53(8)$ & $93(10)$ \\
$\mathrm{C}(71)$ & $174(10)$ & $620(30)$ & $66(5)$ & $96(11)$ & $65(6)$ & $217(16)$ \\
$\mathrm{C}(72)$ & $194(12)$ & $235(15)$ & $163(10)$ & $51(11)$ & $90(9)$ & $29(11)$ \\
$\mathrm{C}(73)$ & $67(6)$ & $134(8)$ & $88(6)$ & $24(6)$ & $20(5)$ & $52(6)$ \\
\hline
\end{tabular}


Table 5. Hydrogen coordinates ( $\left.\times 10^{\wedge} 4\right)$ and isotropic displacement parameters $\left(\mathrm{A}^{\wedge} 2 \times 10^{\wedge} 3\right)$ for $\mathrm{A}$.

\begin{tabular}{|c|c|c|c|c|}
\hline & $\mathrm{x}$ & $\mathrm{y}$ & $\mathrm{z}$ & $\mathrm{U}(\mathrm{eq})$ \\
\hline $\mathrm{HO} 3$ & -3275 & 5321 & 3811 & 80 \\
\hline HO11 & -4699 & -460 & 3485 & 80 \\
\hline $\mathrm{H}(61 \mathrm{~A})$ & -2540 & 7921 & 1005 & 128 \\
\hline $\mathrm{H}(61 \mathrm{~B})$ & -4004 & 6882 & 735 & 128 \\
\hline $\mathrm{H}(61 \mathrm{C})$ & -2675 & 6381 & 770 & 128 \\
\hline $\mathrm{H}(62 \mathrm{~A})$ & -3488 & 3420 & 4664 & 185 \\
\hline $\mathrm{H}(62 \mathrm{~B})$ & -3891 & 2056 & 4207 & 185 \\
\hline $\mathrm{H}(62 \mathrm{C})$ & -5015 & 2798 & 4329 & 185 \\
\hline $\mathrm{H}(63 \mathrm{~A})$ & 263 & 6367 & 2511 & 131 \\
\hline $\mathrm{H}(63 \mathrm{~B})$ & -1232 & 6532 & 2506 & 131 \\
\hline $\mathrm{H}(63 \mathrm{C})$ & -828 & 5856 & 1945 & 131 \\
\hline $\mathrm{H}(64 \mathrm{~A})$ & -2381 & -1904 & 2890 & 137 \\
\hline $\mathrm{H}(64 \mathrm{~B})$ & -2182 & -323 & 3020 & 137 \\
\hline $\mathrm{H}(64 \mathrm{C})$ & -2355 & -1097 & 2387 & 137 \\
\hline $\mathrm{H}(66 \mathrm{~A})$ & -8848 & -2241 & 4016 & 461 \\
\hline $\mathrm{H}(66 \mathrm{~B})$ & -8255 & -2987 & 4461 & 461 \\
\hline $\mathrm{H}(66 \mathrm{C})$ & -8569 & -3641 & 3807 & 461 \\
\hline $\mathrm{H}(67 \mathrm{~A})$ & -4858 & -2071 & 4108 & 186 \\
\hline $\mathrm{H}(67 \mathrm{~B})$ & -5897 & -3511 & 3817 & 186 \\
\hline $\mathrm{H}(67 \mathrm{C})$ & -5716 & -3001 & 4480 & 186 \\
\hline $\mathrm{H}(68 \mathrm{~A})$ & -5909 & -211 & 4590 & 522 \\
\hline $\mathrm{H}(68 \mathrm{~B})$ & -6990 & -1221 & 4862 & 522 \\
\hline $\mathrm{H}(68 \mathrm{C})$ & -7508 & -446 & 4411 & 522 \\
\hline $\mathrm{H}(70 \mathrm{~A})$ & -9593 & 4473 & 4596 & 261 \\
\hline $\mathrm{H}(70 \mathrm{~B})$ & -10742 & 3133 & 4635 & 261 \\
\hline $\mathrm{H}(70 \mathrm{C})$ & -10507 & 3499 & 4036 & 261 \\
\hline $\mathrm{H}(71 \mathrm{~A})$ & -7603 & 4257 & 4947 & 390 \\
\hline $\mathrm{H}(71 \mathrm{~B})$ & -7293 & 2848 & 4926 & 390 \\
\hline $\mathrm{H}(71 \mathrm{C})$ & -8502 & 3131 & 5232 & 390 \\
\hline $\mathrm{H}(72 \mathrm{~A})$ & -9322 & 832 & 4044 & 298 \\
\hline $\mathrm{H}(72 \mathrm{~B})$ & -10305 & 1543 & 3743 & 298 \\
\hline $\mathrm{H}(72 \mathrm{C})$ & -10542 & 1137 & 4335 & 298 \\
\hline
\end{tabular}


Table 6. Hydrogen bonds for A [A and deg.].

\begin{tabular}{lcccc}
\hline D-H...A & d(D-H) & $\mathrm{d}(\mathrm{H} \ldots \mathrm{A})$ & $\mathrm{d}(\mathrm{D} \ldots \mathrm{A})$ & $<(\mathrm{DHA})$ \\
& & & & \\
$\mathrm{O}(3)-\mathrm{HO} 3 \ldots \mathrm{O}(2)$ & $1.091(4)$ & $1.638(5)$ & $2.470(6)$ & $128.6(2)$ \\
$\mathrm{O}(11)-\mathrm{HO} 11 \ldots \mathrm{O}(9)$ & $1.001(4)$ & $1.979(6)$ & $2.883(7)$ & $149.0(3)$ \\
\hline
\end{tabular}

Symmetry transformations used to generate equivalent atoms:

$\# 1-\mathrm{x},-\mathrm{y}-1,-\mathrm{z}$
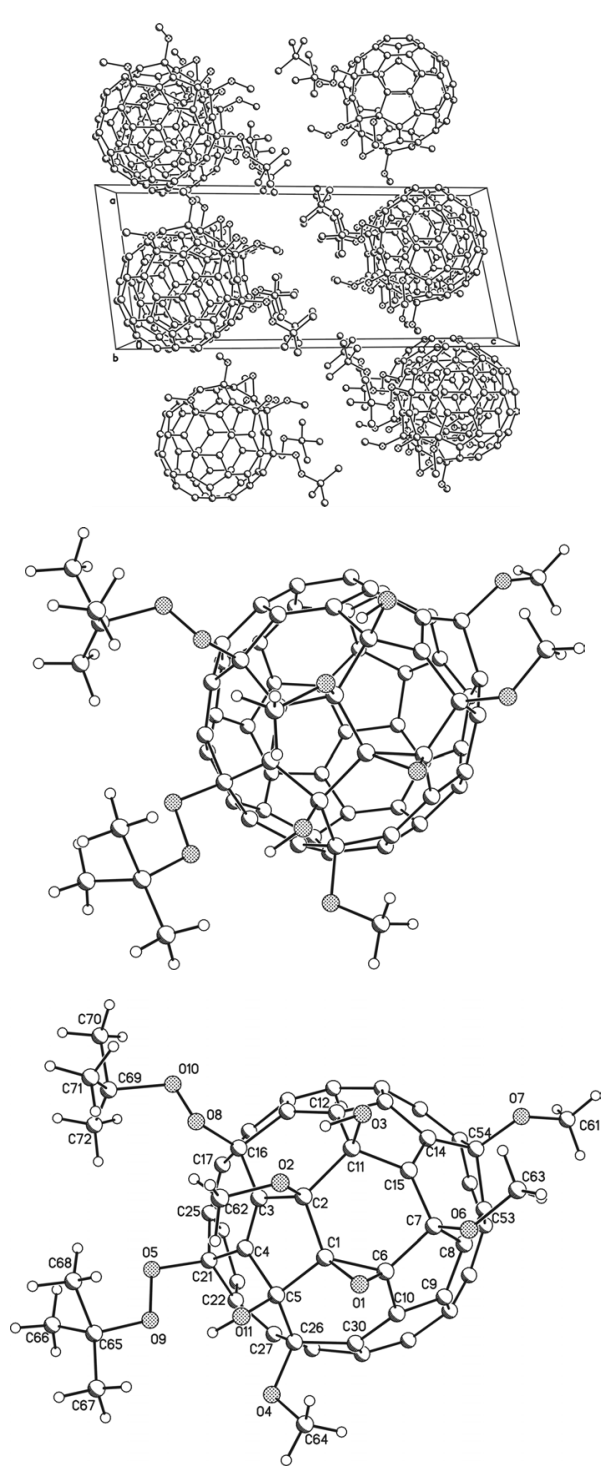